\title{
Frequency recovery techniques for TM/TC satellite modem in critical scenarios
}

\author{
Alessio Fanfani ${ }^{1} \mid$ Simone Morosi ${ }^{1}\left(\mathbb{D} \mid\right.$ Luca Ronga $^{2} \mid$ Enrico Del Re $^{1}$
}

${ }^{1}$ Department of Information Engineering, University of Florence, Via S. Marta 3,

Florence, 50139, Italy

${ }^{2}$ National Inter-University Consortium for Telecommunications, Florence, Italy

\section{Correspondence}

Simone Morosi, Department of Information Engineering, University of Florence, Italy.

Email: simone.morosi@unifi.it

\begin{abstract}
Summary
Reliability and effectiveness are essential features of satellite transceivers for telemetry and telecommand applications. Modem performance has a strong impact on the success of a satellite mission, in particular, during critical scenarios as the early operation phase, the disposal of a satellite at the end of its life, or the deep-space missions. In these specific mission critical scenarios, fast and correct data reception is even more important than high channel capacity. An unknown and fast variable channel condition, which can be caused by uncertain spacecraft attitude and large Doppler shift with respect to the data rate, requires efficient and innovative receiver architecture. This paper introduces a complete digital implementation of a transceiver for TM/TC application in low Earth orbit mission that is perfectly compliant with aforementioned requirements. Particular attention is dedicated to the definition and selection of the most appropriate frequency recovery technique; 2 open-loop techniques that are derived from ML optimal estimator are presented and compared. Additionally, the performance of the proposed receiver is extensively studied and compared with an incoherent technique that is based on the double differential PSK modulation and is known to be suitable for sat-com in critical scenarios.
\end{abstract}

\section{KEYWORDS}

critical scenarios, frequency recovery, satellite modems, telemetry and telecommand applications

\section{1 | INTRODUCTION}

The success of a space mission strongly depends on the quality and performance of satellite subsystems. The Telemetry and Telecommand (TM/TC) is an essential module for a spacecraft along with the electrical power system, on-board data handling and attitude and determination control system. The availability of the communication link with the satellite from the early mission phase allows the ground operator to monitor satellite status and to perform recovery actions if something goes wrong; an on-board computer failure may be recovered by rebooting the associated power line through a direct telecommand to the electrical power system.

The early operations after separation from upper launcher stages, as well as the end of life disposal manoeuvres, are critical satellite mission phases that require a robust and reliable communication system. ${ }^{1,2}$ In these scenarios, the spacecraft may have reduced functionalities and uncontrolled attitude, and consequently, the communication link could be weak and unstable, eg, signal's amplitude may be subjected to deep fading according to satellite tumbling rate. ${ }^{3}$ In this context, the communication becomes bursty and the link reliability harms the bit-rate performance.

Moreover, new challenges are receiving the interest of the space communication community. Nowadays, an innovative and popular way to access space is by means of new low-cost satellite solutions such as micro or nano-satellites. ${ }^{4,5}$ These systems have typically less stringent performance requirements when compared with traditional space systems but, on the other hand, limited energetic and computational resources along with tighter physical constraints.

Taking into account the above-mentioned considerations, this paper proposes a transceiver architecture for TM/TC subsystem in Low Earth Orbit satellite. In particular, it focuses on different solutions for frequency estimation and compensation in presence of Doppler effect, which is one of the main channel impairment in a low Earth orbit (LEO) mission.

The problem of Doppler compensation has been deeply discussed in literature, and many solutions have been proposed, by relying on open-loop 6 or closed-loop ${ }^{7}$ techniques. Noncoherent approach as double differential PSK (DD-PSK) ${ }^{8}$ is attractive due to their system complexity; the lack of a PLL or a carrier acquisition circuit lowers the complexity and the power requirements, but their performance in bit error rate is poor. 
In coherent receivers for LEO application, a frequency recovery circuit is necessary to reduce the offset that is caused by the Doppler shift. A typical frequency recovery system performs 2 functions, the estimation of the carrier frequency offset and the compensation. ${ }^{9}$ When the frequency shift is comparable with the symbol rate, a typical condition during a critical phase, the frequency estimation becomes a challenging task. For instance, the data-aided technique, which is based on the ML-estimation and proposed in one study, ${ }^{10}$ shows performance that is close to the Cramer-Rao lower bound (CRLB) theorethical limit ${ }^{11,12}$ only when the frequency offset is about $20 \%$ of the symbol rate. Alternatively, the estimator proposed in another study ${ }^{13}$ achieves a stable carrier lock after few symbols even for a nonconstant Doppler, but it has an acquisition range that is limited to the $40 \%$ of the symbol rate.

The paper focuses the attention on the open-loop techniques, which are derived from the maximum likelihood estimate under several assumptions and approximations. In particular, it will address 2 different frequency recovery circuits, and their performance will be compared with a receiver implementing an incoherent DD-PSK. ${ }^{14}$ The first solution, which is based on the well-known digital delay and multiply estimator, adopts an innovative configuration consisting of a consecutive coarse and fine estimation and providing a good performance even if the link is affected by a strong Doppler shift, ie, much greater than the bit rate. The other proposed solution takes advantage of the fast Fourier transformation that is used in combination with decimator blocks and filters. This novel implementation reduces the computational effort and the estimation's variance allowing an excellent performance also in critical scenarios that are characterized by fast channel variations. The proposed solutions have been analysed through different numerical simulations, each one dedicated to characterize significant parameters, namely, bias, RMSE, and acquisition time. On the other hand, at system level, the Bit Error Rate has been evaluated by considering theoretical and real link-budget that takes in account time-variant Doppler shift, unstable signal-to-noise ratio, radio frequency impairments such as nonlinearity, and phase noise. The approach addressed in this paper could be used as a reference guideline for satellite communication in critical scenario.

The paper is organized as follows: Section 2 provides an overview of the application's scenario including the link budget in a critical scenario and a characterization of the Doppler shift. Section 3 describes the modem architecture with a focus on the receiver side. Section 4 proposes a complete description of the proposed frequency recovery techniques based on Delay and Multiply and FFT Estimator, an analytical analysis of their operative principles and a simulated comparison of estimator performance. Section 5 compares the performance of the direct-conversion receivers with the incoherent DD-PSK demodulator and shows the simulated results, which have been obtained. Finally, conclusive remarks are given in Section 6.

\section{I SCENARIO}

The target application is a TM/TC radio transceiver for satellite in LEO. This application usually requires low or medium channel rate capability; particularly, a satellite ground operator needs a bit rate of about $32 \mathrm{kbps}$ to control and monitor the satellite within a visibility window. ECSS standards ${ }^{15}$ define the operating conditions and the performance of a typical TM/TC transceiver by requiring that the transceiver shall ensure an essential communication link at all spacecraft attitudes and attitude rates. This condition guarantees that the TM/TC capabilities are available from the first instants of satellite life, launch, and early operation phase, up to the end of the mission, the decommissioning phase.

The operative frequencies that are reserved to TM/TC applications are in the S-band; in particular, the ITU radio regulation and satellite standard ${ }^{16}$ define the following subrange:

- Frequency range: 2025 to $2110 \mathrm{MHz}$ for Earth to space link;

- Frequency range: 2200 to $2290 \mathrm{MHz}$ for space to Earth link.

The reference orbit, which is considered in the study, is a LEO orbit that is similar to the International Space Station one (orbit height and inclination are equal to 450 km and $52 \mathrm{deg}$, respectively).

The target spacecraft is equipped with an hemispherical patch antenna dedicated to TM/TC link that is placed on yaw satellite's face. In nominal satellite condition, with a working attitude stabilization system, this configuration provides a stable signal and the received power is easily computed by means of standard link budget equation:

$$
\frac{E_{b}}{N_{0}}=\frac{C}{N_{0}} \frac{1}{R_{b}}=\operatorname{EIRP}_{T X}\left(\frac{1}{L_{\text {path }} L_{\text {other }}}\right)\left(\frac{G}{T}\right)_{R X}\left(\frac{1}{k}\right) \frac{1}{R_{b}}
$$

where $E_{b} / N_{0}$ is the energy-per-bit to noise-power spectral density ratio, $C / N_{0}$ is the carrier to noise power spectral density ratio, $R_{b}$ is the Bit Rate, $L_{\text {path }}$ and $L_{\text {other }}$ are, respectively, the path loss and the other losses of the link (ie, atmospheric losses and pointing error losses), $G / T$ is the antenna gain over noise temperature ratio, and $k$ is the Boltzmann constant. The selected scenario considers a ground station center equipped with a parabolic dish antenna characterized by an antenna gain up to $35 \mathrm{~dB}$. The link budget of the TM/TC link in the S-band for the selected application is reported in Table 1 ; by assuming a worst case scenario, the lower value of $E_{b} / N_{0}$ is obtained for the telemetry down-link and it is equal to $14.7 \mathrm{~dB}$. It is worth underlining that the channel model does not take in account the effect of multipath fading that in the case of satellite TM/TC link is negligible because the ground station antenna has limited half power beamwidth and features a pointing mechanism. ${ }^{17}$

Figure $1 \mathrm{~A}$ reports the SNR values at the receiver as a function of time during a visibility window; its minimum values are reached at the beginning and at the end of each window, ie, when the satellite is close to the horizon. On the other hand, Figure 1B shows the SNR versus time in case of uncontrolled attitude of the spacecraft; the signal strength presents large variations because of satellite motion. ${ }^{3}$ 
TABLE 1 Link budget table

\begin{tabular}{llcrl} 
Params & \multicolumn{1}{c}{ Description } & $\begin{array}{c}\text { Uplink } \\
\text { worst case }\end{array}$ & $\begin{array}{c}\text { Downlink } \\
\text { worst case }\end{array}$ & Unit \\
\hline$R_{b}$ & Bit Rate & 32768 & 32768 & {$[\mathrm{kbps}]$} \\
\hline$E I R P_{T X}$ & EIRP & 45.2 & 7.0 & {$[\mathrm{~dB}]$} \\
\hline$L_{\text {path }}$ & Path Loss & 165.1 & 165.8 & {$[\mathrm{~dB}]$} \\
$L_{\text {other }}$ & Other Losses [dB] & 17.8 & 17.8 & {$[\mathrm{~dB}]$} \\
$G / T$ & Antenna Gain over Noise Temperature ratio & -27.9 & 10.9 & {$[\mathrm{~dB} / \mathrm{K}]$} \\
$k$ & Boltzmann constant & 228.6 & 228.6 & {$[\mathrm{~dB}]$} \\
\hline$C / N_{0}$ & Carrier to Noise Power Spectral Density raio & 63 & 59.9 & {$[\mathrm{~dB}]$} \\
$E_{b} / N_{0}$ & Energy to Noise Power Spectral Density ratio & 17.8 & 14.7 & {$[\mathrm{~dB}]$} \\
\hline
\end{tabular}

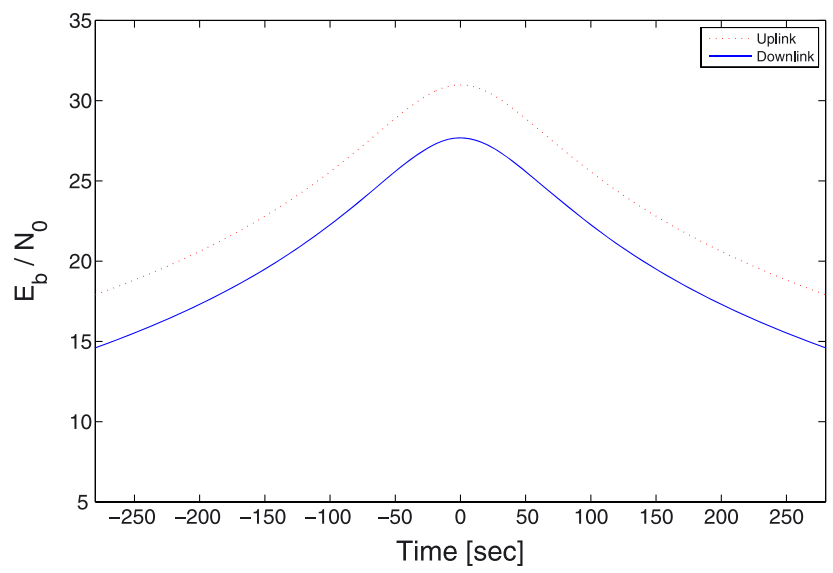

(A) Nominal Attitude Condition

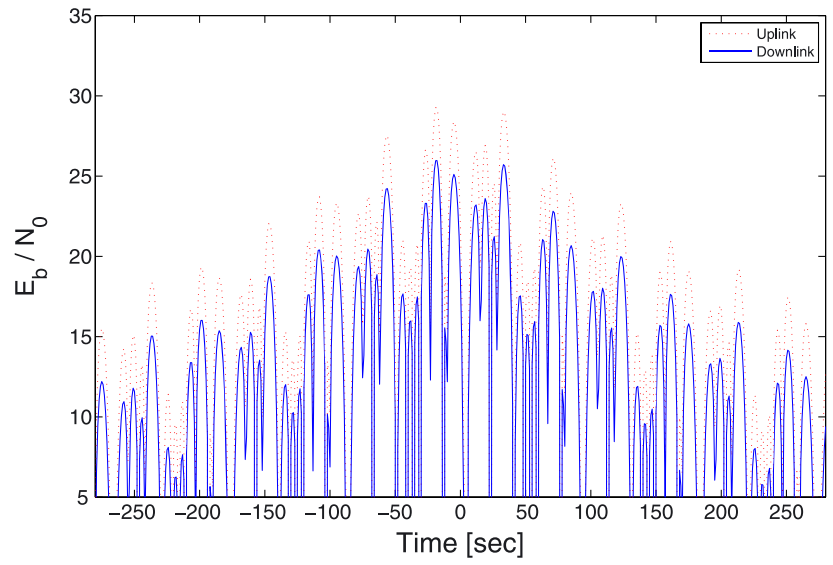

(B) Generic Attitude Condition

FIGURE 1 Link budget curve [Colour figure can be viewed at wileyonlinelibrary.com]

Figure 1B reports the values of the previously considered variables as given by the link budget curves in a possible critical scenario. A high tumble rate has been supposed around all satellite's axes, and the modelled angular rotation velocities are $4 \mathrm{deg} / \mathrm{sec}$ around pitch axis, $14 \mathrm{deg} / \mathrm{sec}$ around Roll axis and $10 \mathrm{deg} / \mathrm{sec}$ around Yaw axis. The depicted sequence of pulses underline the signal instability: particularly, a suitable signal power is obtained only in short burst intervals whose duration is often less than few seconds.

The other main channel impairments in TM/TC link for a satellite that is placed in non-geostationary orbit is the large and time-variant Doppler shift within the visibility window of satellite.

The Doppler effect is caused by the relative motion of the satellite with respect to the ground station. It mainly depends on the satellite orbit, the ground station position (latitude) and the frequency value of the link. The well-known equation of Doppler effect is:

$$
f_{r}=f_{t} \pm \Delta f=f_{t} \pm \frac{v_{r} f_{t}}{c}
$$

where $f_{r}$ is the received signal frequency, $\Delta f$ is the Doppler shift, $v_{r}$ is the radial component of the relative velocity of the receiver with respect to the ground station, and $c$ is the speed of light. 


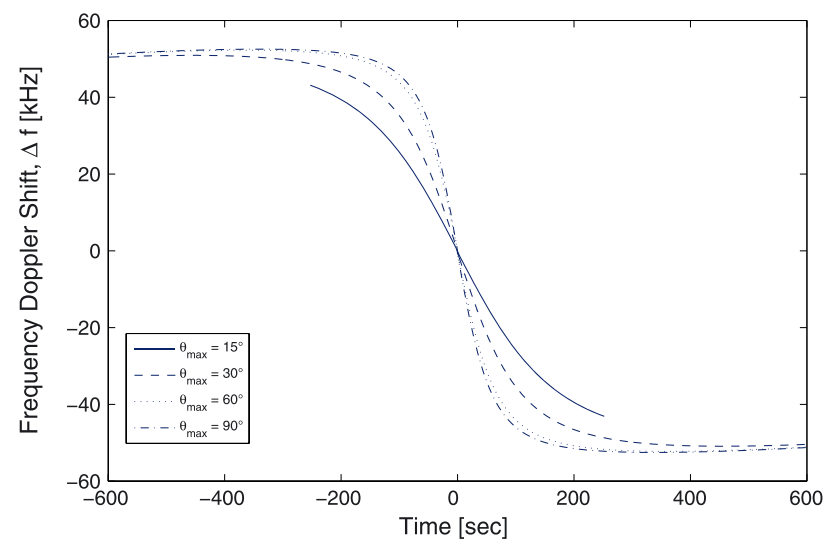

(A) Doppler Shift Variation with in the visibility window

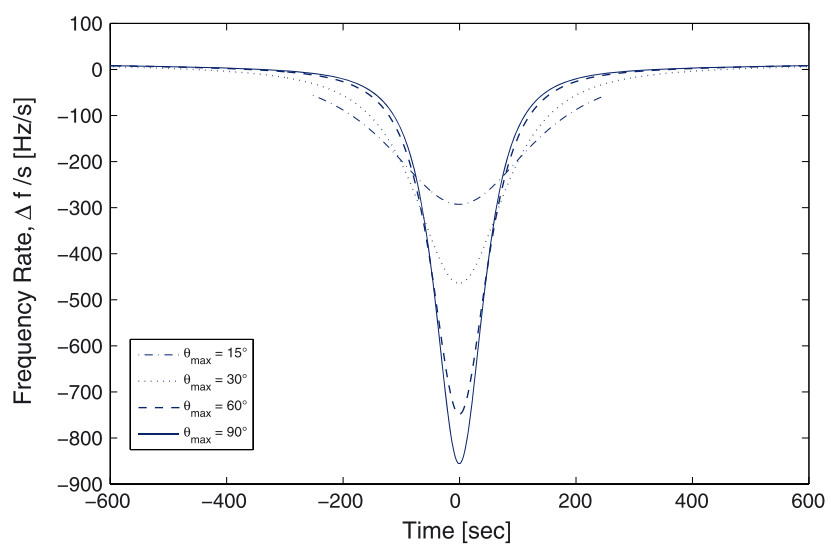

(B) Doppler Rate Variation with in the visibility window

FIGURE 2 Doppler curves [Colour figure can be viewed at wileyonlinelibrary.com]

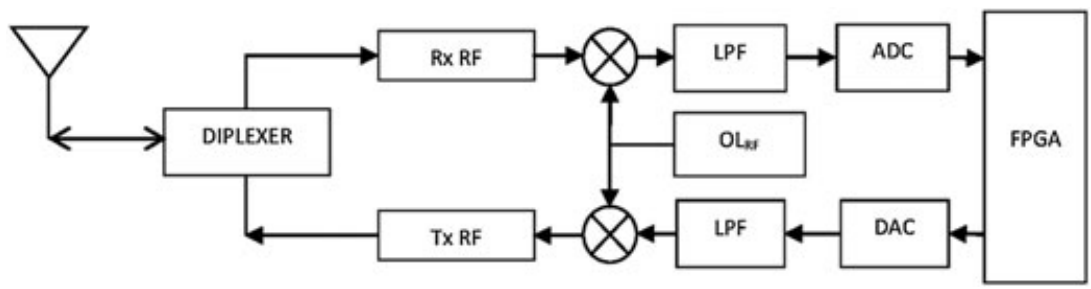

FIGURE 3 Direct-conversion transceiver block diagram

The characterization of Doppler has been largely discussed in literature. In another studies, ${ }^{6,18}$ Ali et al define analytical equations for the Doppler shift and the duration of the satellite visibility windows; these equations are used in the next sections to compare the performance of different receivers in the case of variable Doppler shift.

The frequency Doppler shift is represented by the S-shaped curve, which is shown in Figure 2A considering the above-mentioned ISS orbit; the shift is function of the time and of the maximum elevation angle $\theta_{\max }$; moreover, it is equal to 0 in the middle of the visibility window when the elevation angle reaches the maximum value. The maximum shift is within the $-60 \mathrm{kHz}$ to $60 \mathrm{kHz}$ interval; it occurs when the elevation angle approaches the minimum elevation angle, which is evaluated equal to $10^{\circ}$.

Another important parameter is the Doppler shift rate that represents the shift variation over the time. The curve in Figure 2B describes this feature; the worst case is achieved in the center of visibility window, when the satellite is closer to the ground station, and the Doppler rate is lower than $-800 \mathrm{~Hz}$ per second.

\section{3 | TRANSCEIVER ARCHITECTURE}

The proposed transceiver architecture encompasses an analog front-end with a diplexer to divide the receive and the transmission chains. A high level functional block diagram of the transceiver is depicted in Figure 3. The transceiver uses a direct-conversion implementation, so that the received signal is down-converted to 0-IF frequency and the base band signal is up-converted to the RF band by the radio frequency section. The base band digital section is implemented on a FPGA module. A model of the architecture has been realized in Simulink, so allowing an evaluation of its performance. 


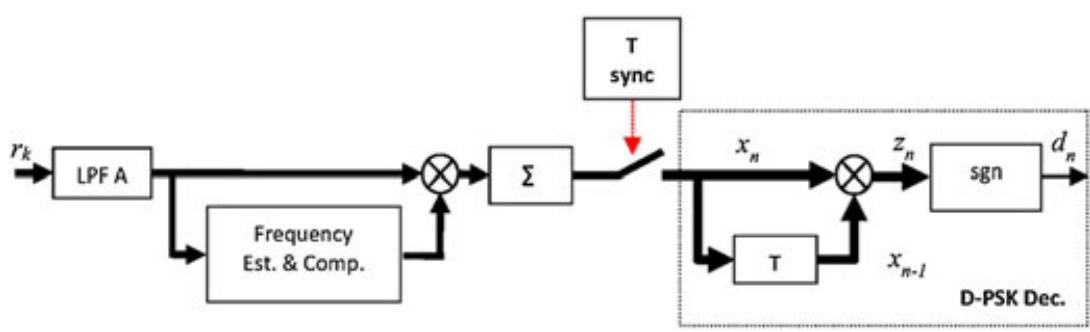

FIGURE 4 Differential PSK (D-PSK) demodulator block diagram [Colour figure can be viewed at wileyonlinelibrary.com]

\section{1 | Transmitter}

The transmitted signal is a differential PSK whose information is found in the phase differences of 2 consecutive bits. The binary data are generated by a random source with bit probability of $50 \%$ and differentially encoded by multiplying the bit with the delayed version of the previous one. For simplicity, we assume that the pulse shape is an ideal rect and satisfies the Nyquist criterion for 0 interference. The analytical form of the D-PSK signal is:

$$
s_{D-P S K}(t)=\sqrt{E_{b}} \cos \left(2 \pi f_{R F} t+\Delta \varphi_{n}\right),
$$

where

- $E_{b}$ is the energy per bit;

- $f_{R F}$ is carrier frequency;

- $\Delta \varphi_{n}=\varphi_{n}-\varphi_{n-1}$ is the differentially coded information; and

- $\varphi_{n}$ is the coded modulation information, i.e. the phase contribution associated to the transmitted data, $\varphi_{n}=n k \pi \rightarrow n \in Z, k=[0,1]$.

\section{2 | Direct-conversion receiver}

The direct-conversion receiver, also known as 0-IF receiver, allows the RF signal to be demodulated by a local oscillator whose frequency is as close as possible to $f_{R F}$, carrier frequency of the received signal. ${ }^{9}$

The strategy that is pursued by the receivers is to estimate the Doppler frequency shift by evaluating the average frequency of the 0-IF input signal. In fact, this signal is frequency shifted away from $0 \mathrm{~Hz}$ by the Doppler effect. After frequency estimation, the Doppler shift is removed by derotating the received signal samples. ${ }^{6}$

In the receiver, the delay and clock recovery is performed before the carrier synchronization; for simplicity, we assumed that the symbol delay is 0 .

The base band digital section is based on the scheme of the D-BPSK demodulator that is depicted in Figure 4: in the diagram, the thick arrows represent the complex-value samples.

The derotating function is performed by a mixer and a direct digital synthesizer, which generates a signal $w_{k}$ that is characterized by Equation 3.

$$
w_{k}=\sqrt{E_{\text {sample }}} \exp \left(-j\left(2 \pi \widehat{\Delta f} T_{\text {sample }} k\right)\right.
$$

The $r_{k}$ signal that is affected by the Doppler shift is mixed with the compensation signals to translate the baseband message signal spectrum closer to the $\mathrm{OHz}$ value. After the frequency compensation, an integrate and dump and a D-BPSK decision complete the receiving chain. The receiver does not need phase recovery scheme before the bit decision because the transmitted signal $r_{k}$ is D-PSK modulated.

The input signal is defined by the following equation:

$$
r_{n}(k)=\sqrt{E_{\text {sample }}} \exp \left(j\left(2 \pi \Delta f T_{\text {sample }} k+\theta_{n}+\Delta \varphi_{n}\right)+n_{k},\right.
$$

where

- $E_{\text {sample }}$ is the energy per sample;

- $T_{\text {sample }}$ is the sampling time;

- $\Delta f$ is the Doppler shift on $n$th symbol;

- $\theta_{n}$ is the carrier phase on $n$th symbol that is assumed to be an unknown random variable uniformly distributed in $[-\pi ; \pi]$;

- $\Delta \varphi_{\mathrm{n}}$ is the differentially coded information of the transmitted signal; and

- $n_{k}$ is a discrete time-complex Gaussian noise (AWGN) with 2-sided power spectral density $N_{0} / 2$. 
TABLE 2 Direct-conversion receiver design parameters

\begin{tabular}{lc} 
Bit rate, $R_{b}$ & $2^{15}=32768 \mathrm{~b} / \mathrm{s}$ \\
\hline Sample frequency, $f_{\text {sample }}$ & $2^{22}=4.194 \mathrm{MHz}$ \\
\hline Maximum doppler shift, $\max \Delta f$ & $60 \mathrm{kHz}$ \\
Low-pass filter bandwidth, $B_{\mathrm{LPF}}$ & $92 \mathrm{kHz}$
\end{tabular}

The $x_{n}$ signal, which is obtained after frequency compensation and filtering, is equal to

$$
\begin{aligned}
x_{n} & =\sum_{k=1}^{128}\left(r_{n}(k) * w_{k}\right)=\sum_{k=1}^{128} E_{\text {sample }} \exp \left(j\left(2 \pi(\Delta f-\widehat{\Delta f}) T_{b} k\right)+\Delta \varphi_{n}\right)+\theta_{n}+n_{k} . \\
& =E_{b} \exp \left(j\left(2 \pi(\Delta f-\widehat{\Delta f}) T_{b} k\right)+\theta_{n}+\varphi_{n}\right)+v_{n} .
\end{aligned}
$$

By assuming a correct estimation, the signal becomes

$$
x_{n}=E_{b} \exp \left(j\left(2 \pi\left(\theta_{n}+\varphi_{n}\right)\right)+n_{k} .\right.
$$

Before analysing the estimator, it is important to observe that the low-pass filter before base band digital section has been designed so that even the signal that is affected by the maximum Doppler shift is allowed to pass. The filter bandwidth is defined by the relation $B_{L P F}=R_{b}+\max \Delta f$; all receiver parameters are reported in Table 2.

\section{4 | DOPPLER SHIFT ESTIMATORS}

The problem of Doppler estimation has been deeply discussed in literature, and many solutions have been proposed. Each solution shows specific pros and cons that make them less or more suitable for the considered applications.

Frequency estimation systems are usually classified according to the values of the acquisition range in comparison with the symbol rate $R_{\text {sym }}{ }^{9}$ :

- when frequency shift is much smaller than the symbol rate (usually when $\Delta f<10 \% R_{\text {sym }}$ );

- when frequency shift is of the order of the symbol rate $R_{\text {sym }}$.

In the first case, data-aided or decision-directed operation could be used with PSK demodulation because timing and data information are computed before frequency recovery.

Data-aided technique based on ML-estimation affords accuracies that are closed to the CRLB ${ }^{11,12}$ for a frequency offset about $20 \%$ of the symbol rate. ${ }^{10}$ In one study, ${ }^{13}$ a close-loop ML estimator that is named "adaptive optimized steepest descent" is described as the best tracking method so far. It provides a stable carrier lock after only 6 symbols also in the case of a nonconstant Doppler; nonetheless, the optimal acquisition range of this system is limited to the $40 \%$ of the symbol rate.

As for the second case, an open-loop ML scheme is proposed in another study, ${ }^{9}$ which is called the delay-and-multiply method. This method is suitable for bursty communications thanks to the very short acquisition time; moreover, it is characterized by a mean square error comparable with the closed-loop techniques. ${ }^{19}$

Finally, it is worth to recall that the ML estimator of the frequency of a sinusoidal signal is the maximum of the periodogram. ${ }^{20}$ Such estimator is theoretically optimum and its analytical formulation is defined by Equation 6

$$
\hat{\Delta} f_{M L}=\arg \max \left|\sum_{k=1}^{N} r_{k} e^{-j \Delta_{f} k}\right|^{2},
$$

where:

- $r_{k}$ represents the samples of the received signal $r(t)$;

- $N$ is the number of available samples; and

- $\Delta_{f}$ is the frequency resolution of the periodogram.

The disadvantage of the maximum likelihood frequency estimator are the excessive computation and storage requirements that are due to its exhaustive search procedures.

An alternative solution is the sweep estimation and compensation technique. ${ }^{21}$ Thanks to the prior knowledge of the transmitter position at the receiver, this technique reduces the Doppler shift by applying a linearly decreasing frequency sweep to the input signal. This technique is not attractive in critical scenario because the prior knowledge of satellite position could not be available.

Two open-loop techniques that are derived from the maximum likelihood estimation have been selected for our application due to their suitability for digital implementation and theoretical optimal performance.

The first solution is an advanced version of the delay-and-multiply estimator. The core of the system is the delay-and-multiply estimator, but this proposal is also influenced by the idea of using a coarse and a fine estimation sequentially as in the receiver realization that is described in another 
study. ${ }^{7}$ The second solution consists of the searching of the maximum of the periodogram through a fast Fourier transformation at a reduced sample rate and after proper filtering process.

\section{1 | Digital D\&M estimator}

The digital delay-and-multiply D\&M estimator, which is represented in Figure 5, matches to the phase variation to determine the instantaneous frequency of the input signal. The rate of change is computed by using the Euler's method of approximating differential equations. ${ }^{22}$ The instantaneous phase is generated by the arctangent of the complex-valued input signal $y_{k}{ }^{7}$ The delay $\Delta T$ of the block that is used to compute the discrete differential equation is equal to the sampling time. The block indicated with the ( $)^{*}$ symbol represents the complex conjugate operator. The digital formulation is defined by the following equation:

$$
\widehat{\Delta f}=\frac{1}{2 \pi \Delta T} \arg \left\{\frac{1}{M} \sum_{k=1}^{M} v_{k}\right\},
$$

where

$$
v_{k}=y_{k} y_{k-1}^{*}=y\left(T_{\text {sample }} k\right) y^{*}\left(\left(T_{\text {sample }}-\Delta T\right) k\right)
$$

The estimator observation time depends on the number $M$ of samples that are used by the estimator. The acquisition range of the D\&M estimator is equal to $\frac{1}{2 \Delta T}$.

The performance of the delay-and-multiply scheme depends on the frequency value of the input signal: the greater the frequency, the greater the variance of the estimation. Hence, the receiver uses 2 delay-and-multiply estimators as shown in Fig. 6: the first one is indicated as A and is a "coarse" estimator working on $r_{k}$; it performs a rough estimation of the frequency shift $\widehat{\Delta f}_{A}$. The $r_{k}$ samples represent a digital and low-pass version of the received signal $r(t)$. A second D\&M is used that is indicated as B: It is a "fine" estimator working on $e_{k}$ signal, that is, the signal with a frequency shift equal to the estimation error of estimation block $A$. Thanks to this mechanism the total estimator has a good performance also for input signals with large frequency Doppler shift. The total estimation, $\widehat{\Delta f_{\text {tot }}}$, is the combination of coarse and fine operations, as defined in Equation 9. The coarse observation time is equal to 128 bits and it is 8 times longer than the "fine" estimation time, that lasts 16 bits.

$$
\widehat{\Delta f_{\text {tot }}}=\widehat{\Delta f_{A}}+\widehat{\Delta f_{B}}
$$

where

$$
\begin{aligned}
& \widehat{\Delta f_{A}}=\frac{1}{2 \pi \Delta T} \arg \left\{\frac{1}{M_{A}} \sum_{k=1}^{M_{A}} z_{k}\right\}, \\
& \widehat{\Delta f_{B}}=\frac{1}{2 \pi \Delta T} \arg \left\{\frac{1}{M_{B}} \sum_{k=1}^{M_{B}} e_{k}\right\} .
\end{aligned}
$$

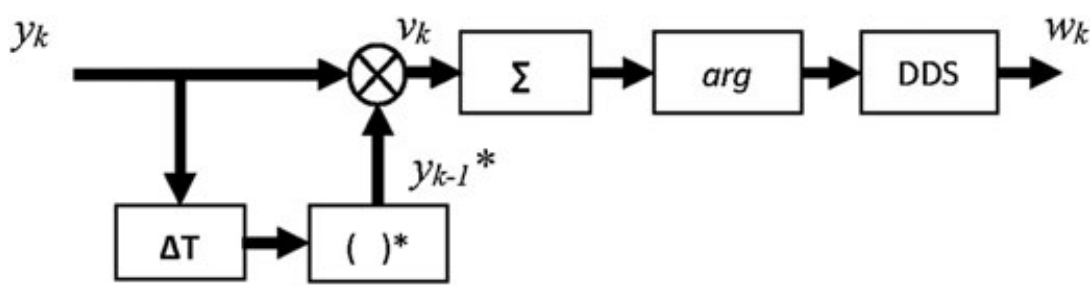

FIGURE 5 Delay-and-multiply 9

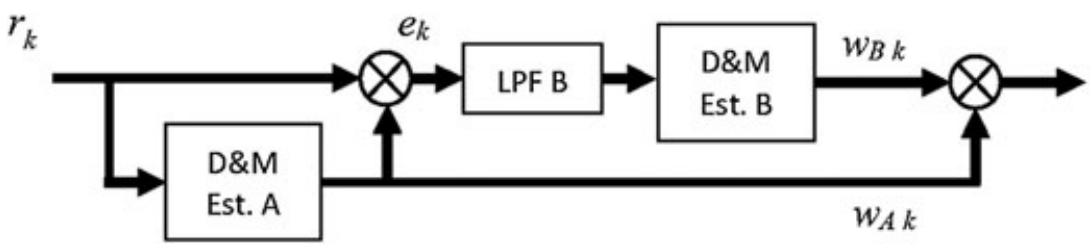

FIGURE 6 D\&M estimator block diagram 
The Figure 7 shows the mechanism of the global D\&M estimator in a scenario that is characterized by $E_{b} / N_{0}=15 \mathrm{~dB}$ and $\Delta f=30 \mathrm{kHz}$. The total frequency estimation needs 2 coarse estimations to converge to the Doppler shift; during the initial 300 bits, the estimation is put equal to 0 due to the initialization of the model.

\section{2 | FFT Estimator}

The second proposed estimator is an improvement of the ML estimator that is based on the the maximum of the periodogram research. The estimator uses the fast Fourier transformation to compute the periodogram and introduces 2 tricks to improve the performance and reduce the computational effort.

Considering that the bandwidth of the input signal of the estimator is the LPF bandwidth, which has been defined in Table 2, the sample rate can be reduced accordingly. For this reason, the first block of the estimator, that is depicted in Fig. 8, is a down-sampler with decimation factor $L=2^{4}=16$ so that the sampling rate becomes $f_{\text {sample }}^{\prime}=2^{18}=262144 \mathrm{~Hz}$. After the down-sampler an FFT is performed by using $N_{F F T}=2^{10}=1024$ samples, resulting in a frequency resolution equal to $256 \mathrm{~Hz}$. The FFT acquisition time expressed in number of bits is computed by Equation 10 .

$$
T_{o}=\frac{N_{F F T}}{f_{\text {sample }}^{\prime}} R_{b}=\frac{2^{10}}{2^{18}} 2^{15}=128 \text { bit }
$$

The second improvement is the addition of an averaging LPF after the FFT block. The effects are described by Equation 11 and depicted in Figure 9 , which shows the signal spectrum before and after the low pass filter; note that the Doppler shift is $30 \mathrm{kHz}$ and $E_{b} / N_{0}=15 \mathrm{~dB}$. The blue curve represents the B-PSK signal spectrum whose maximum is approximately located in correspondence of the Doppler shift while many other peaks

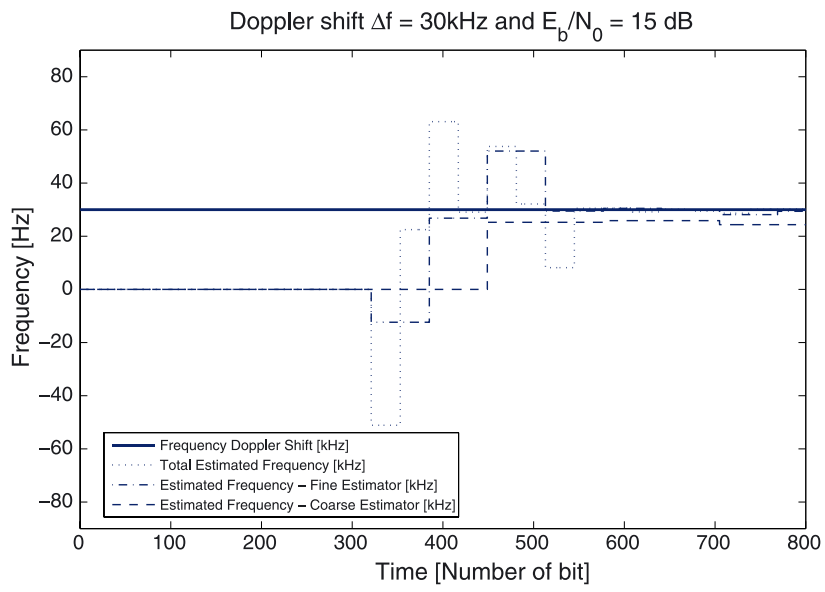

FIGURE 7 Initial frequency acquisition for D\&M estimator, $E_{b} / N_{0}=15 \mathrm{~dB}$ and $\Delta f=30 \mathrm{kHz}$ [Colour figure can be viewed at wileyonlinelibrary.com]

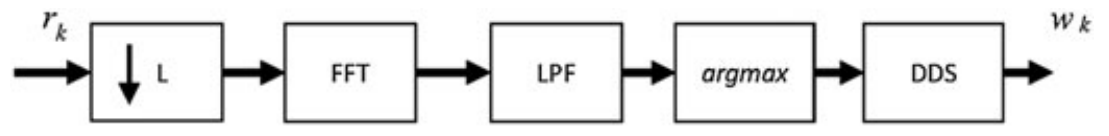

FIGURE 8 FFT Estimator Block Diagram

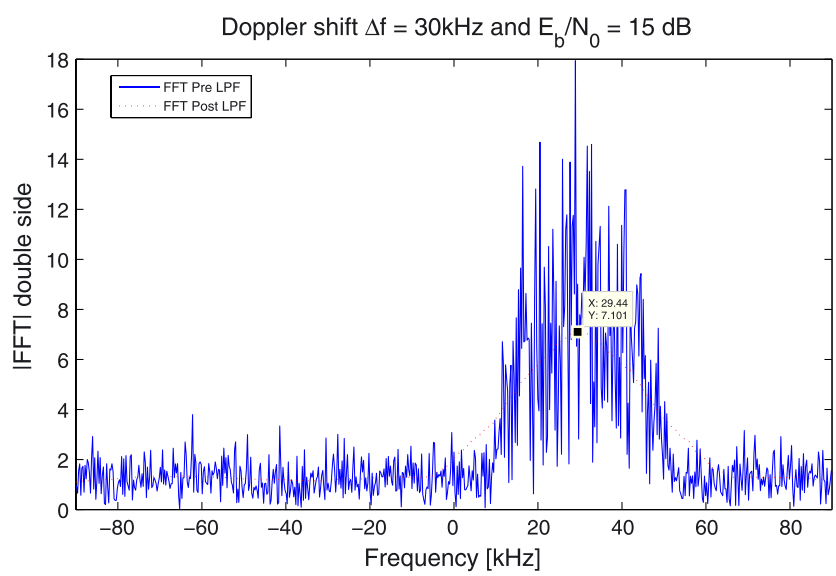

FIGURE 9 FFT signal example, $E_{b} / N_{0}=15 \mathrm{~dB}$ and $\Delta f=30 \mathrm{kHz}$ [Colour figure can be viewed at wileyonlinelibrary.com] 
are placed within the signal bandwidth. The red curve is the low pass filtered version of the blue one; as can be seen, it is a triangular curve with median point corresponding to the Doppler shift. Figure 10 shows the behaviour of estimator during the frequency acquisition phase with (red line) or without (green line) the LPF. The filter improves estimator performance, reducing the variance and stabilizing the estimation. Finally, the FFT estimator analytical formulation is defined by Equation 11.

$$
\widehat{\Delta f}=\operatorname{argmax}\left\{\underline{R}_{F F T} \otimes \frac{\text { ones }\left(N_{F F T}\right)}{N_{F F T}}\right\}
$$

where

$$
\begin{gathered}
\underline{R}_{F F T}=\left[R_{0} R_{1} R_{q} \ldots R_{N-1}\right], \\
R_{q}=\mathcal{F}\{r(k L)\}=\sum_{k=0}^{N_{F F T}-1} r(k L) e^{-i \frac{2 \pi}{N} k L q} \quad q=0,1, \ldots, N-1 .
\end{gathered}
$$

\section{3 | ML estimators comparison}

As it is known, ${ }^{11,12}$ the efficiency of an estimator is inversely proportional to variance of its error, ie, a small variance value is related to good performance. The lowest value of the variance is defined by the CRLB: an unbiased estimator that achieves this lower bound is said to be (fully) efficient. The purpose of this section is to compare the proposed estimators by considering the bias, the variance, and their respective CRLB.

To evaluate the bias, the value of the estimated frequency with respect to the Doppler shift has been computed for different signal-to-noise ratios; the results are shown in Figure 11. The performance of an unbiased estimator should be characterized by an unitary slope; the obtained results testify this feature when $\Delta f$ is up to $60 \mathrm{kHz}$. As expected, the performance degrades for low SNR and when the Doppler shift is greater than the LPF bandwidth $(90 \mathrm{kHz})$.

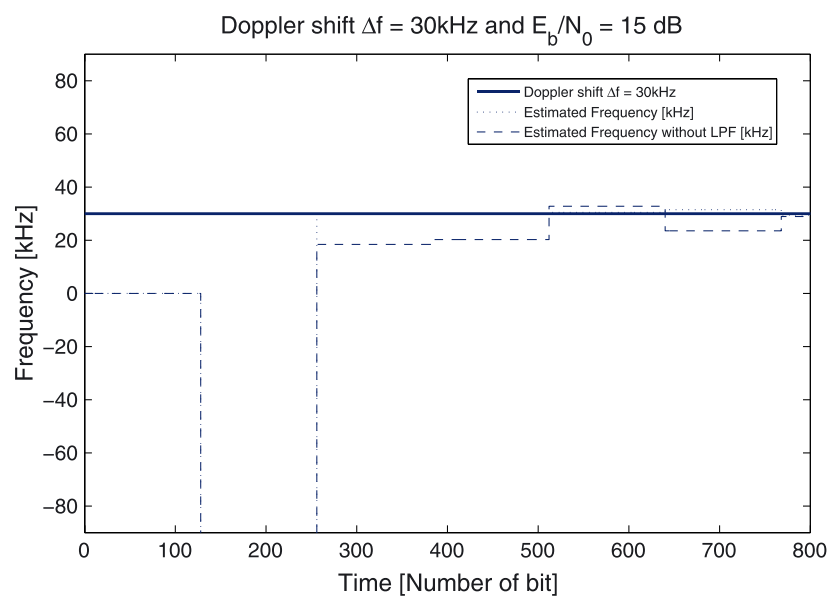

FIGURE 10 Initial Frequency Acquisition for FFT Estimator, $E_{b} / N_{0}=15 \mathrm{~dB}$ and $\Delta f=30 \mathrm{kHz}$ [Colour figure can be viewed at wileyonlinelibrary.com]

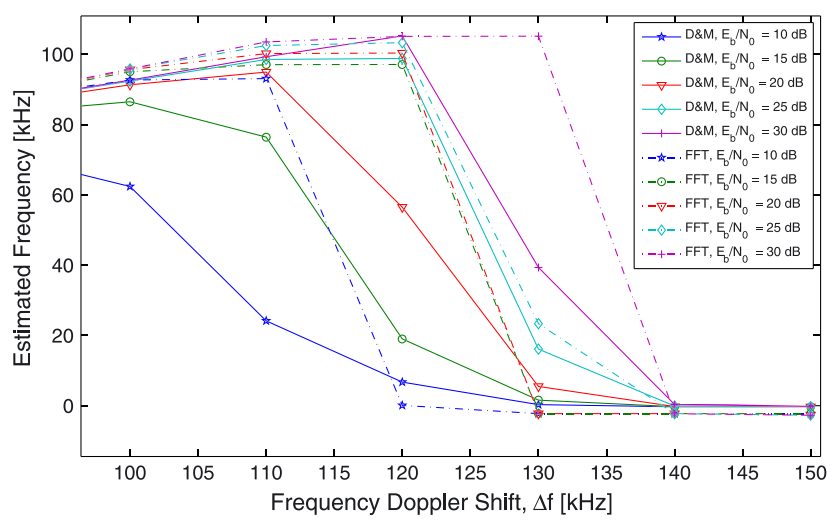

FIGURE 11 Bias for D\&M and FFT estimators for different signal-to-noise ratio [Colour figure can be viewed at wileyonlinelibrary.com] 


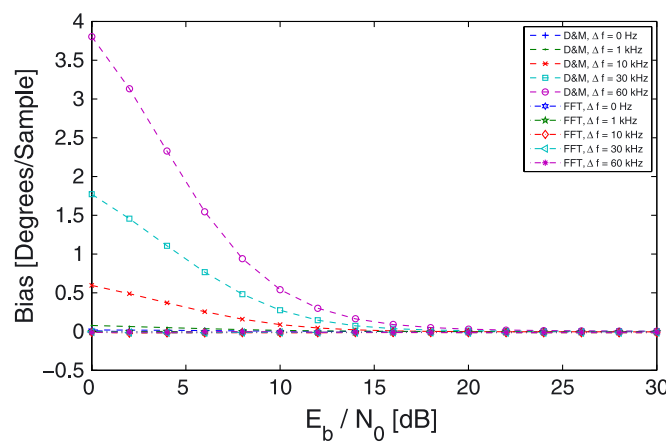

(A) BIAS for different SNR

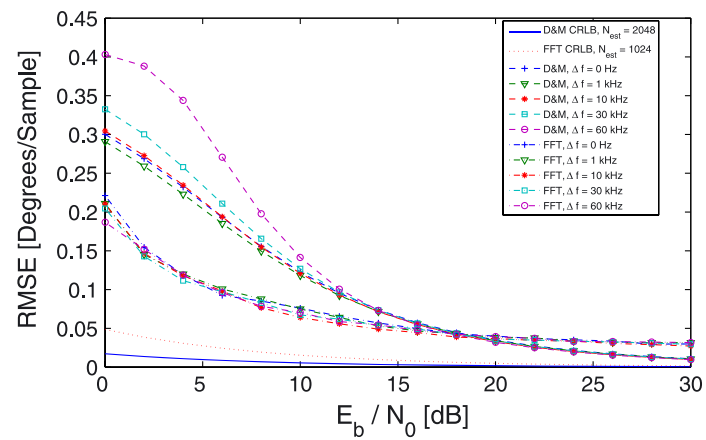

(B) RMSE for different SNR

FIGURE 12 BIAS and RMSE [degrees per sample] [Colour figure can be viewed at wileyonlinelibrary.com]

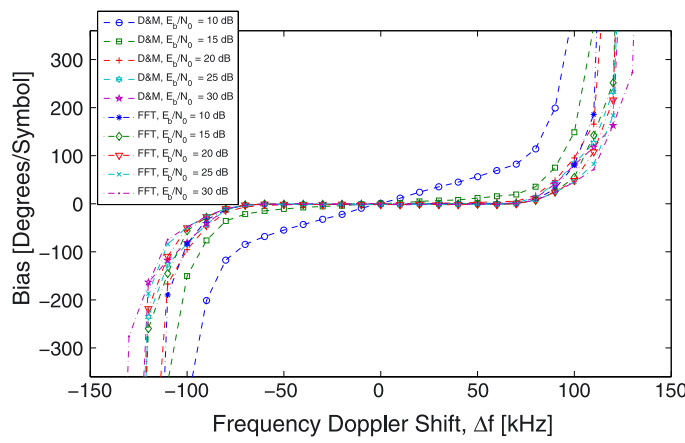

(A) BIAS for different Doppler Shift

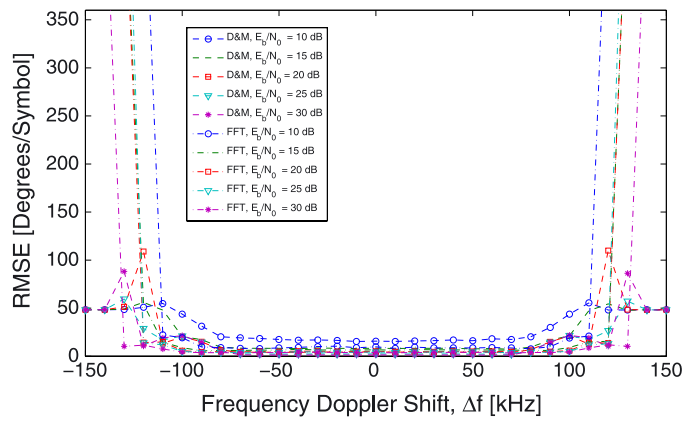

(B) RMSE for different Doppler Shift

FIGURE 13 BIAS and RMSE [degrees per symbol] [Colour figure can be viewed at wileyonlinelibrary.com]

In one study, ${ }^{20}$ Fitz describes a theoretical CRLB of the variance of the frequency estimation that is reported in Equation 12. The formulation uses an estimation error that is defined as $\Omega_{\text {sample }}-\hat{\Omega}_{\text {sample }}=2 \pi(\Delta-\hat{\Delta f}) T_{\text {sample }}$ and $S N R_{b}=128 S N R_{\text {sample }}$.

$$
\begin{gathered}
\operatorname{var}\left(\Omega_{\text {sample }}-\hat{\Omega}_{\text {sample }}\right) \geqslant \frac{6}{N_{\text {est }}\left(N_{\text {est }}{ }^{2}-1\right)} \frac{1}{S N R_{\text {sample }}}, \\
x_{n}=\sqrt{E_{\text {sample }}} e^{j\left(2 \pi \Delta f T_{\text {sample }} n+\theta\right)}+v_{n} .
\end{gathered}
$$

The figures of merits that are used for the comparison of the estimators are

$$
\begin{aligned}
& B I A S \triangleq E\left\{\Omega-\hat{\Omega}_{\text {sample }}\right\}, \\
& R M S E \triangleq \sqrt{\operatorname{var}\left(\Omega-\hat{\Omega}_{\text {sample }}\right)} \geqslant \sqrt{\frac{6}{N_{\text {est }}\left(N_{\text {est }}^{2}-1\right)} \frac{128}{S N R_{b}}} .
\end{aligned}
$$

The parameter $N_{\text {est }}$ is the number of samples that are used for the estimation; the $N_{\text {est }}$ value is equal to 2048 for the D\&M estimator and to 1024 for the FFT based estimator, respectively. Hence, theoretically, the D\&M estimator should show a better performance than the FFT-based one.

The Figure 12 shows the performance of the estimators of BIAS and RMSE over a wide range of possible Doppler shift values, by considering the estimation error, $\Omega_{\text {sample }}-\hat{\Omega}_{\text {sample }}$, over a sample period. The results show that the FFT estimator is almost unbiased and that the D\&M estimator could be considered unbiased only when $E_{b} / N_{0}>15 \mathrm{~dB}$ and $\Delta f>30 \mathrm{kHz}$. Regarding the RMSE, the FFT has a better performance than D\&M in case of low and medium SNR value; when $E_{b} / N_{0}>20 \mathrm{~dB}$, the D\&M converges to the CRLB.

The successive results that are depicted in the graphs of Figure 13 show the performance of the estimators of BIAS and RMSE over a wide range of possible Doppler shift values, by reporting the estimation error over a bit interval rather over a sample period. ${ }^{*}$ Considering that we are using a 


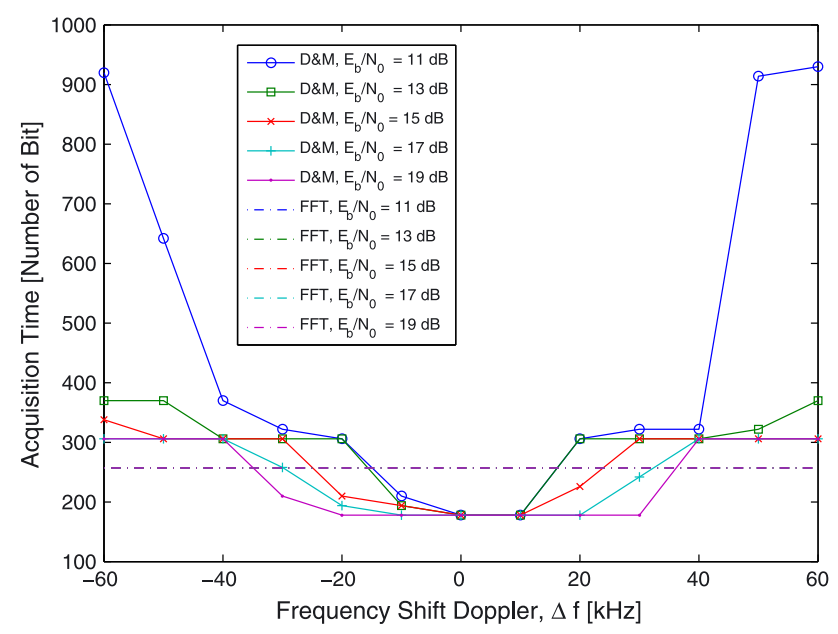

FIGURE 14 Acquisition time comparison [Colour figure can be viewed at wileyonlinelibrary.com]

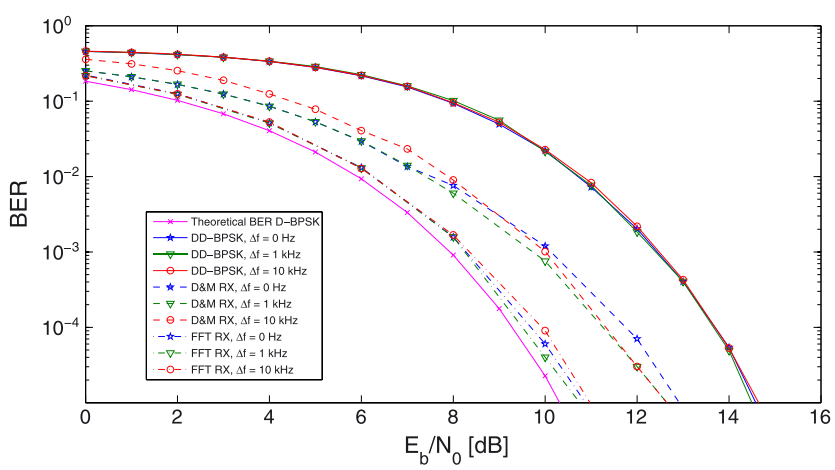

FIGURE 15 Bit error rate for different modulation [Colour figure can be viewed at wileyonlinelibrary.com]

D-BPSK modulation, the maximum acceptable offset error is $90^{\circ}$. The results show again the same conclusion, namely, that the estimator works if the maximum Doppler shift is lower than the low pass filter bandwidth. Again, the FFT-based estimator overcomes the one based on D\&M.

Finally, a comparison of the acquisition time of both receivers for different values of signal-to-noise ratio and Doppler shift has been performed whose results are described in Figure 14. The acquisition time has been defined as the time of number of bits that is requested to keep the phase error caused by the Doppler shift much lower than $\pi$ (ie, $0.1 \pi$ ) where $\pi$ is the phase difference between B-PSK symbols. It is particularly interesting to note that the FFT estimator acquisition time is constant and independent from $E_{b} / N_{0} B$ and $\Delta f$ whereas the D\&M estimator's one increases significantly when the frequency Doppler shift and $E_{b} / N_{0} B$ get worse. Moreover, the D\&M estimator performance is better than the FFT's one if good channel conditions are assumed as under that hypothesis the simple coarse estimation allows to perform the frequency estimation.

\section{5 | SYSTEM COMPARISON}

In this section, we will compare the performance of the estimators when integrated into the receiver; the performance of receivers that are based on incoherent DD-PSK demodulation ${ }^{14}$ will be used as a term of reference. In the following, 3 different scenarios will be considered: the first one, which is called fixed Doppler, is an ideal scenario with constant frequency Doppler shift, ie, no time variation is assumed. The second scenario simulates a link that is affected by a real Doppler shift as defined in Section 2. Finally, the last scenario takes into account an extremely variable signal-to-noise ratio due to the satellite tumbling as the one represented in Figure $1 \mathrm{~B}$ in addition to the Doppler shift.

\section{1 | Fixed Doppler}

The bit error rate curves that are reported in Figure 15 are essentially the same for different Doppler conditions even if the DD-PSK bit error probability is much higher than the other; a better performance of the receiver that is based on delay-and-multiply estimation is clearly highlighted if a time-invariant frequency Doppler shift is assumed. The presented performance can be further improved by the introduction of efficient channel coding techniques. ${ }^{23}$ 
Beyond AWGN and Doppler shifts satellite links can be affected by additional impairments such as the nonlinearity, which is introduced by the high power amplifier and the phase noise that is caused by the RF oscillator; the system's performance with or without the aforementioned radio frequency impairments has been compared, and the results are present in Figure 16. The phase noise impairment has been simulated using as a reference a voltage controlled SAW oscillator ${ }^{24}$ whose performance are reported in Table 3 . The amplification distortion has been modelled using the cubic method of the simulink memory-less nonlinearity block provided by simulink's toolbox. The graphs show that these impairments cause a deterioration of performance of about $2 \mathrm{~dB}$ for both estimators.

\section{2 | Variable Doppler}

This scenario considers a Doppler shift corresponding to the S-curve that is depicted in Figure $2 \mathrm{~A}$ whose relative maximum and minimum elevation angle are equal to $90^{\circ}$ and $20^{\circ}$, respectively. The visibility windows duration is 560 seconds during which about 18 Mbits are transmitted.

The selected S-curve is the worst operative case because it is characterized by the greatest Doppler shift rate in the centre of the visibility windows.

The performance of both the receiving schemes is measured in number of bit errors during a satellite visibility window. The curves in Figure 17 shows the cumulative distribution of bit errors for different values of signal-to-noise ratio. The graphs confirm that direct-conversion receivers have a very robust performance; particularly, the FFT-based estimator results the best one since it has a constant BER during all the visibility time; on the contrary, the DD-PSK performance depends on the Doppler shift rate; particularly, the greatest growing rate of the cumulative distribution is indeed obtained for the maximum value of Doppler rate. In Table 4, the average bit error rates are summarized for different values of signal-to-noise

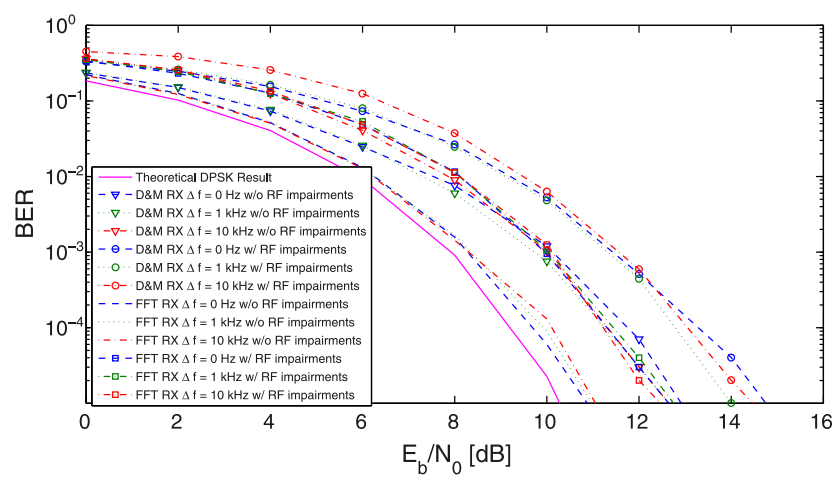

FIGURE 16 Bit error rate with RF impairments [Colour figure can be viewed at wileyonlinelibrary.com]

TABLE 3 Oscillator SSB phase noise 24

\begin{tabular}{ll} 
Phase offset & Phase noise \\
\hline $1 \mathrm{kHz}$ & $-97 \mathrm{dBc} / \mathrm{Hz}$ \\
$10 \mathrm{kHz}$ & $-120 \mathrm{dBc} / \mathrm{Hz}$ \\
$100 \mathrm{kHz}$ & $-140 \mathrm{dBc} / \mathrm{Hz}$ \\
$1 \mathrm{MHz}$ & $-148 \mathrm{dBc} / \mathrm{Hz}$ \\
$10 \mathrm{MHz}$ & $-150 \mathrm{dBc} / \mathrm{Hz}$ \\
\hline
\end{tabular}

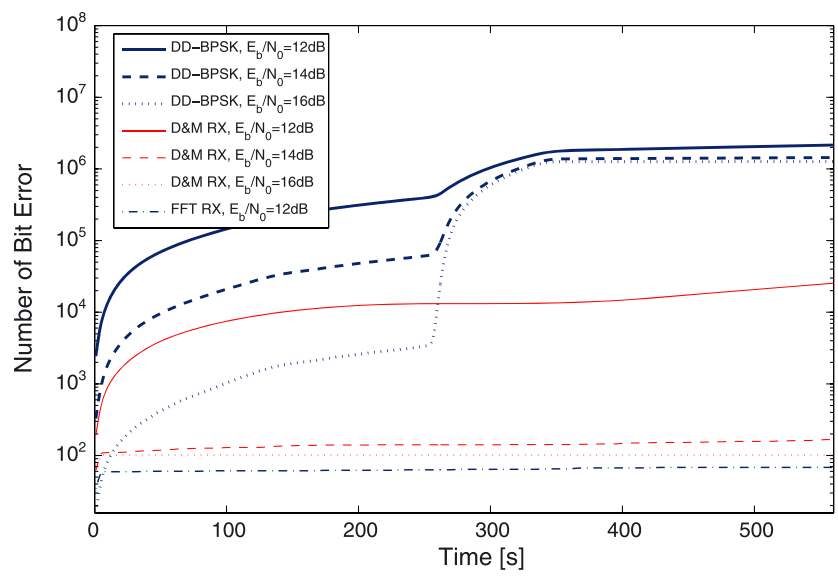

FIGURE 17 Comparison of bit error cumulative distribution for different SNR values [Colour figure can be viewed at wileyonlinelibrary.com] 
TABLE 4 Bit error rate with time variant Doppler shift

\begin{tabular}{lccc}
$E_{b} / N_{0}[\mathrm{~dB}]$ & DD-PSK receiver & D\&M receiver & FFT receiver \\
\hline 12 & $12 \cdot 10^{-2}$ & $1.46 \cdot 10^{-3}$ & $3.76 \cdot 10^{-3}$ \\
14 & $7.9 \cdot 10^{-2}$ & $9.43 \cdot 10^{-6}$ & $3.27 \cdot 10^{-6}$ \\
16 & $6.98 \cdot 10^{-2}$ & $5.61 \cdot 10^{-6}$ & $3.27 \cdot 10^{-6}$ \\
\hline
\end{tabular}

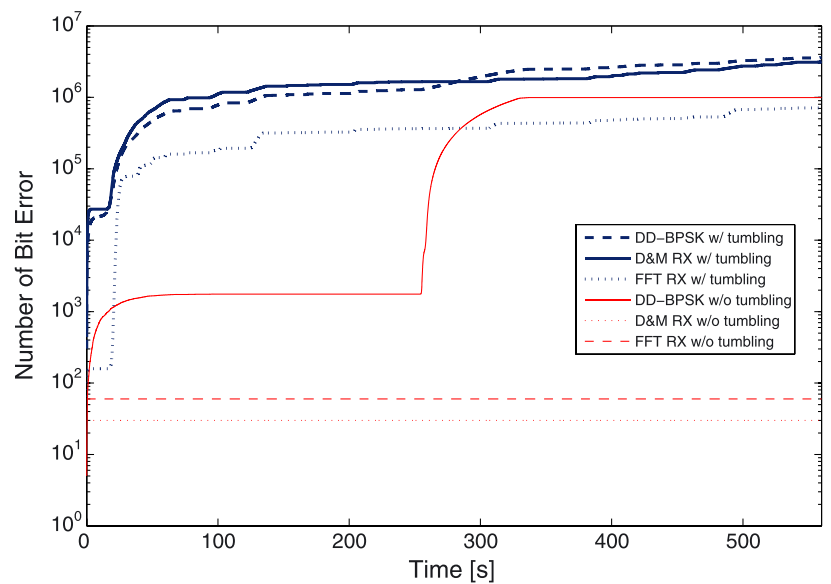

FIGURE 18 Comparison of bit error cumulative distribution within the visibility window [Colour figure can be viewed at wileyonlinelibrary.com]

ratios that are computed at the end of a satellite visibility windows. The BER performance that is obtained by the direct receiver for the variable Doppler shift is similar to the constant Doppler one, so highlighting the effectiveness of the estimator. The BER curves show an initial step due to the wrong bit decoding during the first observation time of the estimators.

\section{3 | Tumbling scenario}

Finally, for sake of completeness, the tumbling satellite scenario is considered. The effectiveness of the receiver that is based on FFT estimator and compensator is also confirmed by the results in Figure 18. The curves represent the bit error cumulative distributions that are achieved over the visibility window; in the sketches, both the situations of Figure 1A,B, namely, a stable and an extremely variable signal-to-noise ratio that is caused by the satellite tumbling, are considered. As expected, in the latter case, the total number of error is very high and the great majority of bit erros are obtained when the $E_{b} / N_{0}$ falls down. As shown by the curves, when the signal quality improves, the bit error cumulative distribution tends to converge to the step function that is depicted in Figure $1 \mathrm{~B}$.

This trend has been also confirmed by comparing the BER for the bits that have been received when the values of $E_{b} / N_{0}$ ratio is greater than $12 \mathrm{~dB}$; in that case, the BER based on FFT and D\&M estimators are, respectively, null and equal to $8 \cdot 10^{-5}$ whereas the DD-PSK BER is equal to 0.1 . This result confirms that the performance of both the D\&M and FFT the estimator is much better than the DD-PSK one.

\section{6 | CONCLUSION}

Space TM/TC applications require reliable and robust modem solutions to guarantee a high performance in critical scenarios, such as in the LEO orbit applications and during the decommission phase when the satellite has random attitude and attitude rates.

In this paper, possible transceiver implementations for the aforementioned applications are proposed and compared; in particular, the paper focus the problem of Doppler shift carrier acquisition for LEO satellites and proposes 2 estimators with an excellent performance and a wide acquisition rage.

The direct-conversion solution has a more complex architecture than the DD-PSK receiver but allows a very good performance. The results show that the D\&M and, in particular, the FFT-based receivers guarantee reliable link also with fast channel variations and strong Doppler shift as shown in this paper.

Both estimators are suitable for implementations for communication in critical scenario as the TM/TC for decommissioning device. The FFT estimator shows a better performance of BIAS and variance than the D\&M. Moreover, the FFT estimator has a smaller observation time, namely, 128 versus 160 bits. 


\section{ACKNOWLEDGEMENT}

This study is the result of a cooperation between the Department of Information Engineering of the University of Florence and D-Orbit, an Italian company that develops a smart satellite device that decommissions satellites at the end of their missions.

\section{REFERENCES}

1. European Code of Conduct for Space Debris Mitigation. June 2004. Issue 1.0.

2. Antonetti S, Ferrario L, Toson E, Rossettini L. Implementation of Debris Prevention and Reduction Measures by an Independent Propulsive Decommissioning Device. In: 66th International Astronautical Congress. Jerusalem; 2145-2155, 12-16, October 2015.

3. Bruzzi JR, Jensen JR, Fielhauer KB, Royster DW, Srinivasan DK. Telemetry recovery and uplink commanding of a spacecraft prior to three-axis attitude stabilization. In: Proceedings of the 2006 IEEE Aerospace Conference. Big Sky, MT, United States of America; 1-11.

4. Toson E, Fanfani A, Morosi S, Ronga LS, Martegani P, Rossettini L. D-Sat Mission: An In-Orbit Demonstration of a solid propellant satellite decommissioning device. In: The 4S Symposium 2016. Valletta, Malta; 1-9.

5. Ronga LS, Jayousi S, Pucci R, Morosi S, Berioli M, Rammer J, Fanfani A, Antonetti S. Multiple Alert Message Encapsulation Protocol: Standardization and Experimental Activities. In: Proceedings of the ISCRAM 2015 Conference - Kristiansand, May 24-27 - Palen, Bscher, Comes \& Hughes. Kristiansand; Norway; $1-10,2015$.

6. Ali I, Bonanni PG, Al-Dhahir N, Hershey JE. Doppler Application In LEO Satellite Communication Systems. New York, Published by Springer US. ISBN $9780306475467,1997$.

7. van der Westhuizen E, van Rooyen G-J. Baseband Carrier Recovery and Phase Tracking as a Doppler Compensation Technique for a zero-IF SDR. In: Proceedings of the Southern African Telecommunication Networks and Applications Conference. Royal Swazi Spa, Swaziland; 1-6, 2009.

8. Yuce MR, Wentai L, Damiano J, Bharath B, Franzon PD, Dogan NS. SOI CMOS Implementation of a multirate PSK demodulator for space communications. IEEE Transactions on Circuits and Systems I: Regular Papers. 2007;54(2):420-431.

9. Mengali U, D'Andrea AN. Synchronization Techniques for Digital Receivers. New York, Published by Springer Science \& Business Media, 2013. ISBN 1489918078.

10. Mengali U, Morelli M. Data-Aided Frequency Estimation for Burst Digital Transmission. IEEE Transactions on Communications. 1997;45(1):23-25. https:// doi.org/10.1109/26.554282

11. Proakis JG, Salehi M. Digital Communications (5th). New York, Published by MacGraw-Hill. ISBN $0072321113,2007$.

12. Kay SM. Fundamentals of Statistical Signal processing: Estimation Theory. Prentice Hall: Upper Saddle River, NJ; 1993.

13. George Patrick Ah-Thew. Doppler Compensation for LEO Satellite Communication Systems. Ph.D. dissertation, 1998.

14. Del Re E, Fanfani A, Morosi S, Ronga LS. Robust Modem Design for Satellite Communications in Emergency Scenarios, 2014 7th Advanced Satellite Multimedia Systems Conference and the 13th Signal Processing for Space, Sept. 2014.

15. ECSS-E-ST-50-05C Rev. 2. Radio Frequency and Modulation, ESA-ESTEC - 4, 2011.

16. CCSDS 401.0-B. Radio Frequency and modulation system, 2013.

17. Maral G, Bousquet M. Satellite Communications Systems: Systems. Techniques and Technology. Pearson Education India.

18. Ali I, Al-Dhahir N, Hershey JE. Doppler characterization for LEO satellites. IEEE Trans Commun. 1998;46(3):309-313.

19. Classen adn F, Meyr H. Two Frequency Estimation Schemes Operating Independently of Timing Information. In Conf. Rec. GLOBECOM'93. Houston, TX, November 29 -December 2, 1993; 1996-2000.

20. Fitz MP. Further results in the fast estimation of a single frequency. IEEE Trans Commun. 1994;42(234):862-864.

21. El-Hennawey MS, Carter CR. Total information processor for 121.5/243 MHz SARSAT signals. IEEE Trans Aerosp Electron Syst. $1992 ; 28: 866-877$.

22. Franklin GF, David Powell J, Workman M. Digital Control of Dynamic Systems (3rd edn.) Addison Wesley Longman, Inc.: Menlo Park, CA; 1998.

23. Lin S, Costello DJ. Error Control Coding. Englewood Cliffs, New Jersey, Pearson Education India, 2005.

24. Technical Datasheet - Spectrum Microwave: MN725-2500.0000 Revision 100909.

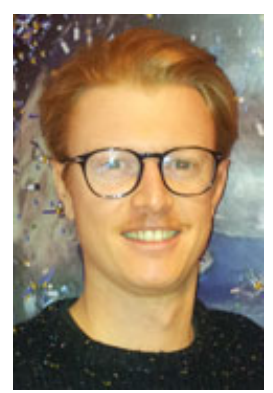

Alessio Fanfani received his MS degree in telecommunication engineering in 2013 and his PhD degree in telecommunications in 2017 from the University of Florence, Italy. Since 2013, he worked on D-Orbit D-SAT mission as System Engineer and later as Program Manager: As System Engineer, he was in charge of the satellite design, integration, verification, and testing. He also had the responsibility for design and implementation of a ground station for satellite application operative in VHF/UHF bands. The main research area of interest is Satellite telecommunication services for applications in emergency scenarios. He's an author of several transaction and conference papers. 


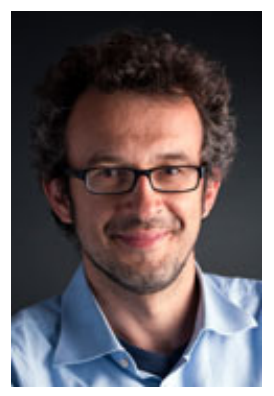

Simone Morosi received the DEng Degree in Electronics Engineering and the PhD Degree in Information and Telecommunication Engineering from the University of Florence, Florence, Italy, in 1996 and 2000, respectively. Since 1999, he has been a researcher at the Italian Interuniversity Consortium for Telecommunications (CNIT). He has been with the Electronics and Telecommunications Department of the University of Florence since 2000 as a Research Scientist, since November 2006 as an Assistant Professor. Since 2013, he has been an Assistant Professor with the Engineering Information Department of the University of Florence where he gives lectures about Digital Signal Processing systems. His present research interests include Green ICT, Wireless and Satellite Communications and Positioning techniques. He has been involved in several National Research projects with task leadership responsibilities. He has published more than 30 papers in international journals and more than 100 conference contributions.

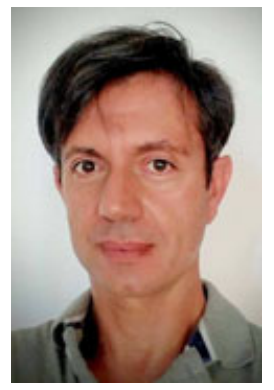

Luca Ronga received his MS degree in electronic engineering in 1994 and his PhD degree in telecommunications in 1998 from the University of Florence, Italy. In 1997, he joined the International Computer Science Institute of Berkeley, California, as a visiting scientist. In 1999, he joined the Italian National Consortium for Telecommunications, where he is currently the head of research area. His research activities, proven by over 120 published papers in international journals and conferences, range from signal processing and physical layer to networking and data transport issues. He has been an editor of EURASIP Newsletter for 4 years, member of ETSI SatEC working group and author of ETSI standards, and member of NATO task force on Cognitive Radio and Software defined Radio. His current research interests are focused on software-defined networking, security for radio communications, and satellite systems.

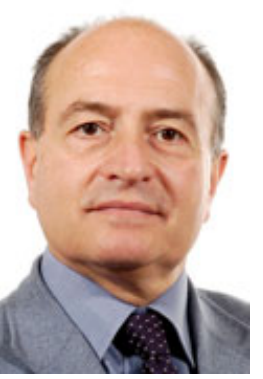

Enrico Del Re received his degree in Electronic Engineering from the University of Pisa, Italy, in 1971. Since 1975, he joined the Department of Electronics and Telecommunications Engineering of the University of Florence where he is currently the Full Professor of Digital Signal Processing. From 2001 to 2016, he has been a member of the Executive Board of CNIT (Italian University Consortium for Telecommunications). He is the head of the Signal Processing and Communications Laboratory of the Department of Information Engineering (DINFO) of the University of Florence. The professional and academic experience has been focused in the fields of the telecommunications systems and the digital processing of signals, where he has published 6 books, more than 200 papers in national and international journals and conferences and more than 30 technical reports. He has been responsible of over 50 research contracts with the Italian National Research Council, Italian Space Agency, European Union, and industries. He has been responsible of a research contract with the European Space Agency in 1984 to 1986 on the application of advanced digital signal processing techniques on board the satellites. He has been the Chairman of the EU COST Projects 227 "Integrated Space-Terrestrial Mobile Networks" and 252 "Evolution of satellite personal communications from second to future generation systems."

How to cite this article: Fanfani A, Mo rosi S, Ronga L, Del Re E. Frequency recovery techniques for TM/TC satellite modem in critical scenarios. Int J Satell Commun Network. 2018;36:179-193. https://doi.org/10.1002/sat.1211 